\title{
UM MODELO DIFERENCIAL PARA HISTERESE MAGNÉTICA: REPRESENTAÇÃO ALGÉBRICA RECURSIVA
}

\author{
Luiz Alberto L. de Almeida* \\ lalberto@ufba.br \\ Antonio Marcus N. Lima ${ }^{\dagger}$ \\ amnlima@dee.ufcg.edu.br
}

\author{
Gurdip S. Deep ${ }^{\dagger}$ \\ deep@dee.ufcg.edu.br \\ Helmut Neff ${ }^{\dagger}$ \\ hn@vir-tech.dk
}

\begin{abstract}
*Departamento de Engenharia Elétrica - Universidade Federal da Bahia - R. Aristides Novis, 2 - Federação CEP 40210-630 - Salvador - BA - Brazil

†Departamento de Engenharia Elétrica - Universidade Federal de Campina Grande - Caixa Postal 10105 CEP 58109-970 - Campina Grande - PB - Brazil

${ }^{\ddagger}$ VIR-TECH A/S, Kuldyssen 10, DK-2630 Taastrup, Denmark
\end{abstract}

\begin{abstract}
The usually employed macroscopic models for magnetic hysteresis are the Jiles and the Preisach models. When incorporated in computer-aided design tools (CAD) for electromagnetic analysis, these models require numerical approximation methods that impose a certain computational burden in the hysteresis calculations. Many analysis applications are very sensitive to this burden, particularly the combination of magnetic hysteresis with finite element method. Further, to analyze real devices using these CAD tools, it is necessary to first adjust the model to experimental data. For these models, the available parameter determination procedures require many steps to correctly fit the model to experimental data. This paper introduces a simple algebraic model to describe magnetic hysteresis. With only four parameters, it has low computational burden and reduced mathematical complexity, permitting thus a fast numerical implementation and simple parameter estimation procedure. The proposed model is also presented in its differential form, and a comparison of its mathematical
\end{abstract}

\footnotetext{
Artigo submetido em 20/12/00

1a. Revisão em 11/04/02

Aceito sob recomendação do Ed. Assoc. Prof. Edson H. Watanabe
}

structure with those of the Jiles and the Preisach models is described. Simulation results are presented and the model performance is discussed in terms of its capacity to represent common nonlinearities associated with the magnetic hysteresis phenomenon. A MATLAB script for numerical implementation of the model, describing the magnetic hysteresis in a MnZn power ferrite is presented.

KEYWORDS: magnetic hysteresis, computational magnetics, finite elements, magnetic losses, minor loop, major loop, accommodation, Madelung rules, magnetization, rate-independent model.

\section{RESUMO}

Os modelos de Jiles e Preisach são os modelos macroscópicos de histerese magnética mais utilizados atualmente. Quando incorporados em programas de computador para auxílio a projeto e análise (CAD), estes modelos requerem métodos numéricos que impõem uma certa carga computacional no cálculo da histerese. Muitas aplicações de análise são sensíveis a esta carga computacional, em especial a combinação do método de elementos finitos com a histerese magnética. Para anali- 
sar dispositivos reais utilizando estas ferramentas CAD, é necessário que o modelo seja ajustado aos dados experimentais. Para os modelos de Jiles e Preisach, os procedimentos disponíveis para determinação dos parâmetros, a partir de dados experimentais, são trabalhosos. Neste artigo é proposto um modelo algébrico simples para descrever a histerese magnética. Com apenas quatro parâmetros, este modelo possui baixa carga computacional e complexidade matemática reduzida, permitindo assim uma implementação numérica de execução rápida e procedimento simples de estimação de parâmetros. O modelo proposto é também apresentado na sua forma diferencial e uma comparação entre a estrutura matemática do mesmo e as correspondentes estruturas dos modelos de Jiles e Preisach é descrita. Resultados de simulação são apresentados e o desempenho do modelo é discutido em termos da capacidade do mesmo em representar não-linearidades comuns da histerese magnética. Um script MATLAB para implementação numérica do modelo, descrevendo a histerese magnética em ferrites de potência tipo MnZn é apresentado.

PALAVRAS-CHAVE: Magnetic hysteresis, computational magnetics, finite elements, magnetic losses, minor loop, major loop, accommodation, Madelung rules, magnetization, rate-independent model.

\section{INTRODUÇÃO}

O termo histerese está relacionado com a memória em uma propriedade de um sistema ou material, cujo comportamento independe da taxa de variação da excitação com o tempo (Visintin, 1994). Isto significa que a curva de histerese é estável e sua memória tem persistência temporal. Entretanto, grande parte dos materiais que exibem histerese, a exemplo de plasticidade, ferroeletricidade e ferromagnetismo, não é puramente independente da taxa de excitação. De fato, existe um efeito viscoso ou inercial nos fenômenos de histerese (Ohta et al., 1991). Quando a taxa de variação da grandeza de excitação tende a zero, o efeito de dependência com a taxa se torna muito pequeno e a histerese pode ser considerada independente da taxa.

O fenômeno de histerese inclue: a forma e o desenvolvimento de laços principais e parciais; a forma e a existência de laços menores; o processo de acomodação em laços menores (Torre, 1994), congruência e deleção (Mayergoyz, 1991); e outros efeitos derivados de excitação estocástica (Bertotti, 1999).

Diversos modelos têm sido propostos para descrever a histerese, cada um deles com características mais adequadas para descrever uma certa classe de materiais. $\mathrm{O}$ modelo de Prandtl-Reuss permite representar diversos fenômenos de atrito e viscosidade, e é um modelo contínuo de histerese dos mais elementares (Visintin, 1994). O modelo de Preisach, originalmente proposto para ferromagnetismo (Torre, 1999)(Bertotti, 1999), é capaz de descrever diversos tipos de histerese (Brokate e Sprekels, 1996). O procedimento proposto em (Mayergoyz, 1991) para implementar numericamente o modelo de Preisach, em conexão com dados experimentais de materiais ferromagnéticos, foi responsável por tornar este modelo aceito e aplicado em diversos fenômenos. O modelo de Krasnoselskii-Pokrovskii, ou modelo K-P, é uma generalização do modelo de Preisach através da introdução de um operador contínuo, em contraste com o operador elementar descontínuo deste último (Krasnoselskii et al., 1989). Um modelo diferencial para histerese magnética, baseado em considerações de energia, foi originalmente proposto em (Jiles e Atherton, 1983), o qual tem alguma semelhança matemática com o modelo estudado por Duhem (Brokate e Sprekels, 1996). Uma abordagem tipo "caixa-preta" para modelagem da histerese magnética, utilizando redes neurais, tem recebido considerável atenção nos últimos anos (Saliah e Lowther, 1997). Entretanto, este tipo de modelo caixa-preta ainda necessita de alguma componente que possui memória persistente, a exemplo do operador tipo play (Serpico e Visone, 1998).

No contexto dos princípios físicos fundamentais, há vários mecanismos envolvidos no processo de formação da histerese magnética (Bertotti, 1999): magnetização de rotação, movimento das paredes de domínios e nucleação. Estes mecanismos estão presentes simultaneamente na maioria do materiais magnéticos, em proporções diferentes e não necessariamente independentes uns dos outros. É extremamente difícil isolar os efeitos destes mecanismos e tratá-los de maneira independente. Não obstante esta superposição de causas, a modelagem da histerese magnética pode ser realizada estabelecendo-se certa conexão com alguns destes mecanismos físicos (Jiles e Atherton, 1983). Por outro lado, em um nível fenomenológico, as fórmulas matemáticas propostas são desenvolvidas para representar o comportamento experimental observado, sem qualquer relação com os princípios físicos envolvidos (Hodgdon, 1988; Mayergoyz, 1991). Além disso, algumas modificações podem ser feitas nos modelos fenomenológicos, com base nos princípios físicos, para tornar estes modelos mais precisos (Torre, 1991; Basso e Bertotti, 1996; Basso et al., 1998).

Neste artigo é proposto um modelo fenomenológico para histerese em materiais magnéticos, com uma estrutura de memória diferente daquelas presentes nos modelos 
de Jiles e Atherton (1983) e Preisach (1935). O modelo proposto é apresentado em termos de uma equação algébrica com apenas quatro parâmetros, não sendo necessária a solução de equações integro-diferenciais. As componentes reversíveis e irreversíveis da magnetização estão incluídas no modelo. As não-linearidades mais comuns do fenômeno de histerese são descritas e, para situar o modelo proposto no arcabouço comum deste tipo de modelagem, o mesmo é comparado com os modelos de Jiles e Preisach. Para este objetivo, os três modelos são apresentados na forma de equação diferencial, permitindo que as principais características destes modelos sejam apontadas e comparadas.

\section{TERMINOLOGIA DO FENÔMENO DE HISTERESE}

Num material que exibe histerese, é necessário que sejam distinguidas duas grandezas experimentais: a excitação $x$ e a resposta $y$. Freqüentemente, os termos sinal de entrada e sinal de saída são também utilizados para $x$ e $y$, respectivamente.

A não-linearidade ou característica mais conhecida da histerese é o laço principal. Para obtenção do laço principal, o valor de $x$ é inicialmente elevado até que a resposta $y$ atinja a parte reversível da curva ${ }^{1}$. Deste ponto, o valor de $x$ é reduzido até que a resposta $y$ atinja a outra parte reversível da curva. A parte do laço principal para a qual $\frac{d y}{d t}<0$ é chamada de curva decrescente principal. Inversamente, a parte do laço principal para a qual $\frac{d y}{d t}>0$ é chamada de curva crescente principal. Se em qualquer ponto das curvas decrescente e crescente principais o sinal de $\frac{d x}{d t}$ muda, a trajetória no plano $x-y$ se aproxima respectivamente das curvas crescente e decrescente principais. Este ponto é chamado de ponto de reversão, e a trajetória que emana do mesmo é denotada de curva de reversão de primeira ordem. Se em qualquer ponto da curva de reversão de primeira ordem ocorre um outro ponto de reversão, a trajetória resultante é chamada de curva de reversão de segunda ordem, e assim por diante. A trajetória fechada, resultante de duas curvas de reversão consecutivas, é chamada de laço menor. A seqüência de trajetórias abertas, formadas por curvas de reversão consecutivas, é chamada de laços menores aninhados. Na prática, os laços menores não formam trajetórias completamente fechadas, mesmo quando sujeitos a uma excitação com valores compreendidos entre os mesmos extremos. Isto implica que, a formação dos laços menores é precedida de um processo de estabiliza-

\footnotetext{
${ }^{1} \mathrm{~A}$ região da histerese considerada como reversível é aquela na qual, para um determinado valor de excitação, só há um único valor de resposta. Para materiais magnéticos, esta região normalmente corresponde à saturação.
}

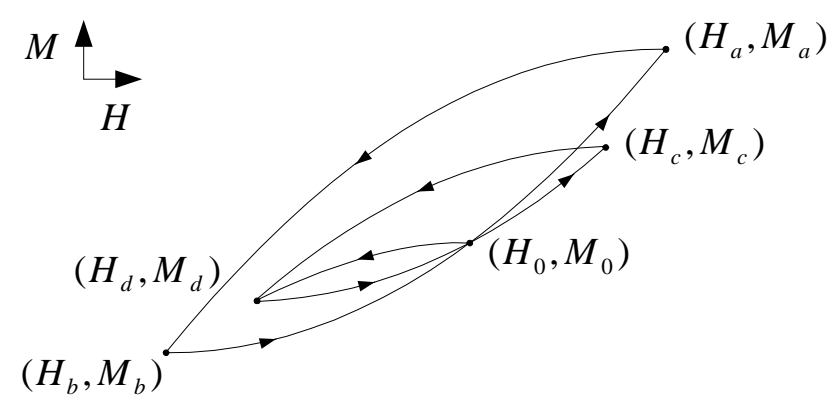

Figura 1: Ilustração das propriedades de memória relativas ao fechamento de laços menores, remoção de extremos de reversão e memória não-local.

ção conhecido como acomodação (Torre, 1994).

\section{PROPRIEDADES DA HISTERESE MAGNÉTICA}

Em um material magnético, o estado atual $\left(H_{0}, M_{0}\right)$ em um instante arbitrário $t=t_{0}$ pode assumir qualquer valor dentro do laço limite principal, dependendo da história da excitação $H(t)$ para $t<t_{0}$. No caso da histerese independente da taxa, não é necessário o conhecimento de todos os valores passados de $H(t)$ para prever $M\left(t_{0}\right)$, mas apenas alguns valores extremos do passado. Para identificar estes extremos relevantes, são descritas a seguir duas propriedades de memória definidas para o fenômeno de histerese.

A primeira delas é a propriedade de retorno ao ponto de partida (return-point memory), descrita como segue. Se a trajetória originando de $\left(H_{a}, M_{a}\right)$ é revertida em $\left(H_{b}, M_{b}\right)$, o novo trecho retorna a $\left(H_{a}, M_{a}\right)$ (Figura 1$)$. Na prática, se o campo varia entre $H_{a}$ e $H_{b}$, o laço menor desloca-se até um laço de equilíbrio e a propriedade de retorno ao ponto de partida não é perfeitamente válida (Torre, 1994).

A segunda propriedade está relacionada com o aspecto de memória não-local, conforme segue. Para melhor descrever esta propriedade, considera-se duas trajetórias alternativas para chegar ao mesmo estado $\left(H_{d}, M_{d}\right)$, dadas por

$$
\left(H_{a}, M_{a}\right) \searrow\left(H_{b}, M_{b}\right) \nearrow\left(H_{0}, M_{0}\right) \searrow\left(H_{d}, M_{d}\right)
$$

$\mathrm{Ou}$

$$
\left(H_{c}, M_{c}\right) \searrow\left(H_{d}, M_{d}\right)
$$

sendo que $\nearrow \mathrm{e} \searrow$ representam campos em crescimento e decrescimento monotônicos, respectivamente. Uma trajetória de histerese originando do ponto de reversão 


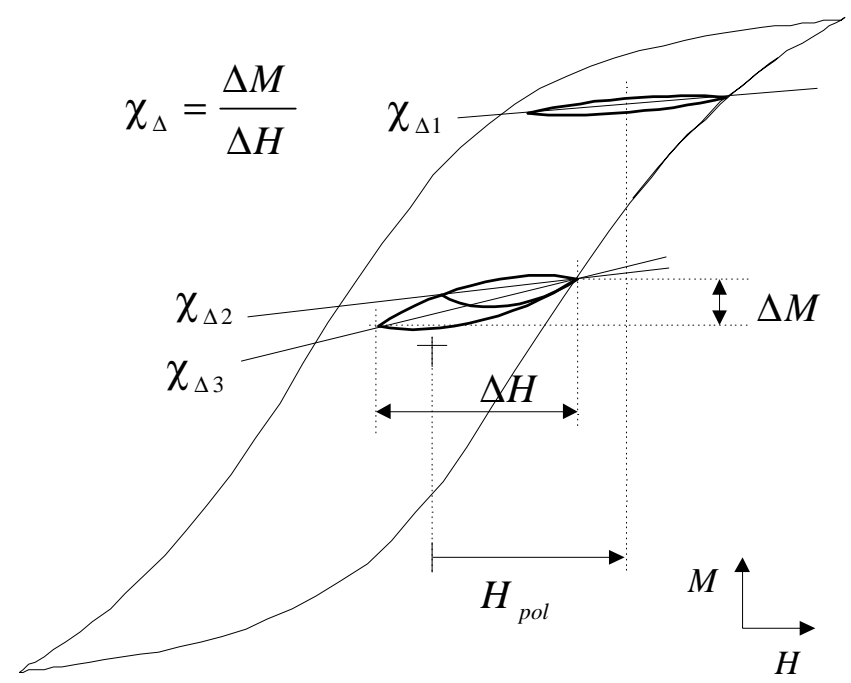

Figura 2: Susceptibilidade Incremental.

$\left(H_{d}, M_{d}\right)$ e passando por $\left(H_{0}, M_{0}\right)$, pode bifurcar em duas trajetórias diferentes

$$
\left(H_{d}, M_{d}\right) \nearrow\left(H_{0}, M_{0}\right) \nearrow\left(H_{a}, M_{a}\right)
$$

ou

$$
\left(H_{d}, M_{d}\right) \nearrow\left(H_{0}, M_{0}\right) \nearrow\left(H_{c}, M_{c}\right)
$$

dependendo se o estado $\left(H_{d}, M_{d}\right)$ foi alcançado através de (1) ou (2), respectivamente.

Outra característica interessante da histerese magnética é o comportamento de desmagnetização do material. Esta desmagnetização é obtida aplicando no material um campo magnético com amplitude alternada decrescente, para produzir um conjunto de laços com larguras progressivamente menores. Uma curva de magnetização iniciando neste estado de desmagnetização $(M=0, H=0)$ é chamada de curva virgem de desmagnetização, na qual a susceptibilidade inicial não é necessariamente nula. Se o processo de desmagnetização é realizado aplicando um campo magnético alternado decrescente com um certo valor médio, o estado final resultante é um ponto na curva sem histerese (anhysteretic curve).

Um campo alternado de baixa amplitude $\Delta H$ com valor médio $H_{\text {pol }}$, produz uma mudança correspondente na magnetização com amplitude $\Delta M$ (Figura 2). A razão $\chi_{\Delta}=\frac{\Delta M}{\Delta H}$ é chamada de susceptibilidade incremental. $\mathrm{O}$ valor de $\chi_{\Delta}$ varia com o campo $H_{\text {pol }}$, decrescendo à medida em que $H_{\text {pol }}$ aumenta.

\section{MODELO ALGÉBRICO}

É conhecido que, na característica experimental de histerese $M \times H$, as curvas crescente e decrescente principais são separadas por uma certa largura. A magnetização $M$ satura para valores elevados negativos e positivos de $H$. Além disso, todas as trajetórias de histerese estão confinadas ao laço limite, que é a combinação das curvas crescente e decrescente principais (curvas limite).

É proposta uma função $F_{L}(H)$ para descrever a magnetização $M$ nas curvas limite crescente e decrescente, na forma

$$
M=F_{L}(H) \triangleq \frac{2}{\pi} M_{s} \arctan \left(\frac{H-\delta H_{c}}{h_{0}}\right),
$$

sendo $M_{s}$ a magnetização de saturação, $H_{c}$ o campo coercitivo, $h_{0}$ uma constante dependente do material e $\delta=\operatorname{sgn}(\dot{H})$.

A combinação das curvas $F_{L}(H, \delta=+1)$ e $F_{L}(H, \delta=$ -1) resulta na descrição matemática do laço principal no plano $M-H$, e representam a região na qual todas as possíveis trajetórias de histerese podem estar (Figura 3).

Utilizando a equação (3), não é possível descrever laços menores, laços aninhados e curvas crescentes ou decrescentes de primeira ordem. Esta equação deve ser modificada para também representar a dependência de $M$ com $H$ para qualquer trajetória dentro do laço principal. Assim, para introduzir esta modificação, propõe-se levar em consideração a forma com a qual uma determinada trajetória dentro do laço principal se aproxima das curvas limite crescente e decrescente. Este conceito é ilustrado na figura 3 , sendo a trajetória $(k-1)$ revertida no ponto $\left(H_{r}, M_{r}\right)$ do qual uma nova trajetória $k$ começa.

O campo de proximidade $H_{p}$ é definido para expressar a distancia entre o ponto atual $\left(H_{0}, M_{0}\right)$ em uma trajetória $k$ ao ponto correspondente $\left(H_{L}, M_{0}\right)$ na curva limite $F_{L}(H)$, dado por

$$
H_{p} \triangleq H_{L}-H_{0}
$$

Utilizando (3), o valor de $H_{L}$ é obtido para o ponto correspondente $M_{0}=F_{L}\left(H_{L}\right)$ como

$$
H_{L}=h_{0} \tan \left(\frac{\pi M_{0}}{2 M_{s}}\right)+\delta H_{c} .
$$

Assim, $H_{p}$ em $\left(H_{0}, M_{0}\right)$ é obtido a partir de (4) como sendo

$$
H_{p}=h_{0} \tan \left(\frac{\pi M_{0}}{2 M_{s}}\right)+\delta H_{c}-H_{0} .
$$




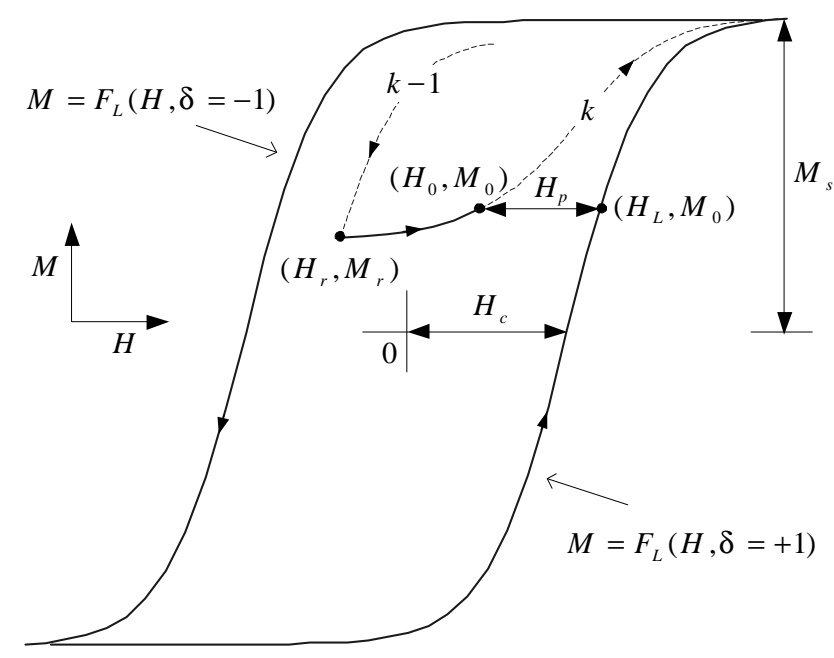

Figura 3: Representação esquemática da histerese na característica $M \times H$. Esta construção geométrica de uma trajetória após a reversão ilustra o conceito de proximidade da mesma ao laço principal, que é formado pela combinação das curvas limite $F_{L}(H, \delta=+1)$ e $F_{L}(H, \delta=-1)$.

No começo de uma nova trajetória, no ponto de reversão $\left(H_{r}, M_{r}\right)$, o campo de proximidade $H_{p}$ é denotado de $H_{p r}$ e resulta em

$$
H_{p r}=h_{0} \tan \left(\frac{\pi M_{r}}{2 M_{s}}\right)+\delta H_{c}-H_{r} .
$$

Da observação de dados experimentais, é possível estabelecer que a dependência funcional de $H_{p}$ para qualquer trajetória dentro do laço principal é quase independente do ponto de reversão $\left(H_{r}, M_{r}\right)$. Assim, a seguinte dependência funcional é proposta para descrever $H_{p}$

$$
H_{p} \triangleq H_{p r} P(x)
$$

sendo $x=\frac{H_{0}-H_{r}}{H_{p r}}$ e $P(x)$ uma função arbitrária monotonicamente decrescente, com $P(0)=1$, denotada de função de proximidade.

Substituindo (7) em (5) resulta em

$$
H_{p r} P(x)=h_{0} \tan \left(\frac{\pi M_{0}}{2 M_{s}}\right)+\delta H_{c}-H_{0},
$$

e a magnetização correspondente ao ponto atual $\left(H_{0}, M_{0}\right)$ pode ser obtida de (8) e escrita como

$$
M_{0}=\frac{2 M_{s}}{\pi} \arctan \left(\frac{H_{p r} P(x)+H_{0}-\delta H_{c}}{h_{0}}\right) .
$$

Da equação (9), para qualquer ponto arbitrário $(H, M)$, a dependência da magnetização $M$ com o campo $H$ é dada por

$$
M(H)=\frac{2 M_{s}}{\pi} \arctan \left(\frac{H_{p r} P\left(\frac{H-H_{r}}{H_{p r}}\right)+H-\delta H_{c}}{h_{0}}\right) .
$$

Os valores de $\delta, H_{r}$ e $H_{p r}$ mudam apenas nos pontos de reversão, e permanecem inalterados até que ocorra uma nova reversão em $H(t)$. É interessante observar a simplicidade do modelo, que requer apenas os parâmetros $M_{s}, h_{0}, H_{c}$ e $\zeta$.

A função de proximidade depende do tipo de material magnético, e necessita ser escolhida com critério para que se ajuste adequadamente à dependência de $H_{p}$ com $H$. Para um material magnético tipo suave, propõe-se a seguinte função de proximidade

$$
P(x) \triangleq\left\{\begin{array}{cc}
1-\sin \zeta x, & \zeta x<\frac{\pi}{2} \\
0, & \zeta x>\frac{\pi}{2}
\end{array}\right.
$$

sendo $\zeta$ uma constante arbitrária.

\subsection{Procedimento de Identificação de $\mathrm{Pa}$ - râmetros}

A Eq.3 pode ser ajustada ao laço principal da histerese experimental, desde que este laço tenha sido obtido excursionando-se o campo $H$ até que a magnetização atinja saturação total. Para identificar corretamente $M_{L}(H, \delta)$, é necessário que sejam determinados 3 parâmetros: $M_{s}, H_{c}$ e $h_{0}$. O valores de $H_{c}$ e $M_{s}$ podem ser extraídos diretamente por inspeção dos dados experimentais de um laço principal com saturação plena.

Não é possível extrair o valor de $h_{0}$ direto de inspeção dos dados experimentais. Para encontrar $h_{0}$ é necessário um procedimento de ajuste de curva, que leva em consideração tanto o trecho ascendente $\left.\tilde{M}_{L}\right|_{\delta=+1} ^{H=H_{s a t}^{-} \ldots H_{s a t}^{+}}$ quanto o trecho descendente $\left.\tilde{M}_{L}\right|_{\delta=-1} ^{H=H_{s a t}^{+} \cdots H_{s a t}^{-}}$do laço principal experimental. Estes trechos experimentais devem ser ajustados às curvas $F_{L}(H, \delta=+1)$ e $F_{L}(H, \delta=$ $-1)$, respectivamente. Considerando que os parâmetros $H_{c}$ e $M_{s}$ estão já estimados, o valor de $h_{0}$ pode ser determinado resolvendo-se o seguinte problema de otimização

$$
\widehat{h_{0}}=\underset{h_{0} \in \mathbf{R}}{\arg \min } \sum_{H=H_{\text {sat }}^{-}}^{H_{\text {sat }}^{+}}\left\{\begin{array}{c}
{\left[F_{L}(H, \delta=-1)-\left.\tilde{M}_{L}\right|_{\delta=-1}\right]^{2}} \\
+\left[F_{L}(H, \delta=+1)-\left.\tilde{M}_{L}\right|_{\delta=+1}\right]^{2}
\end{array}\right\} .
$$

Uma vez que os parâmetros da função $F_{L}(H, \delta= \pm 1)$ estejam determinados, é possível determinar o valor de 
$\zeta$. Entretanto, para obter o valor de $\zeta$, é necessário gerar uma curva experimental decrescente de primeira ordem (first-order descending curve - FOD) $\left.\tilde{M}_{F O D}\right|_{\delta=-1} ^{H=H_{i} \ldots H_{\text {sat }}^{-}}$ (de Almeida et al., 2000)(Mayergoyz, 1991). Uma curva FOD é gerada primeiro reduzindo o campo $H$ para o seu valor máximo negativo $H_{\text {sat }}^{-}$, no qual a magnetização atinge a saturação negativa $M_{s a t}^{-}$. No próximo passo, o campo $H$ é monotonicamente aumentado, até que atinja um certo valor $H_{i}$ com correspondente magnetização $M_{i}$. Neste momento, é possível gerar os valores da FOD, sendo $\left(M_{i}, H_{i}\right)$ seu ponto inicial, e a magnetização $M_{F O D}(H)$ medida com o campo sendo reduzido monotonicamente até o valor $H_{\text {sat }}^{-}$. A escolha da FOD deve ser tal que permita um ajuste adequado do parâmetro $\zeta$. A curva experimental $\left.\tilde{M}_{F O D}\right|_{\delta=-1} ^{H=H_{c} \ldots H_{\text {sat }}^{-}}$é então traçada iniciando-se no ponto $M_{i}=0$ e $H_{i}=H_{c}$. Esta curva deve então ser ajustada à curva $M(H, \delta=-1)$ gerada pelo modelo (10) para a mesma excitação. Isto é equivalente à solução do seguinte problema de otimização

$$
\widehat{\zeta}=\underset{\zeta \in \mathbf{R}}{\arg \min } \sum_{H=H_{c}}^{H_{\text {sat }}^{-}}\left[M(H, \delta=-1)-\left.\tilde{M}_{F O D}\right|_{\delta=-1}\right]^{2},
$$

completando dessa forma o procedimento de identificação dos parâmetros do modelo.

\section{COMPARAÇÃO ENTRE OS MODE- LOS: ALGÉBRICO, JILES E PREISACH}

No modelo proposto por Jiles, a magnetização total $M$ é obtida como uma soma das componentes irreversível $M_{i r r}$ e reversível $M_{r e v}$ de magnetização. A componente reversível é uma função descrita por uma função semelhante a $F_{L}(H, \delta=0)$ e a componente irreversível é representada por uma equação diferencial não-linear

$$
\frac{d M_{i r r}}{d H}=f(H, M, \delta) \text {. }
$$

O modelo de Jiles inclui informação sobre a história da excitação $H$ através apenas do estado atual $\left(H_{0}, M_{0}\right)$. O modelo que possui esta característica é usualmente denominado de modelo de histerese com memória local.

Em contraste com o modelo de Jiles, o modelo clássico de Preisach inclui informação sobre a história da excitação através do armazenamento dos valores extremos passados de $H$. Estes valores estão associados ao modelo de Preisach através de uma estrutura discreta de memória chamada de seqüência de memória (Visintin, 1994), que correspondem aos vértices internos do triangulo de

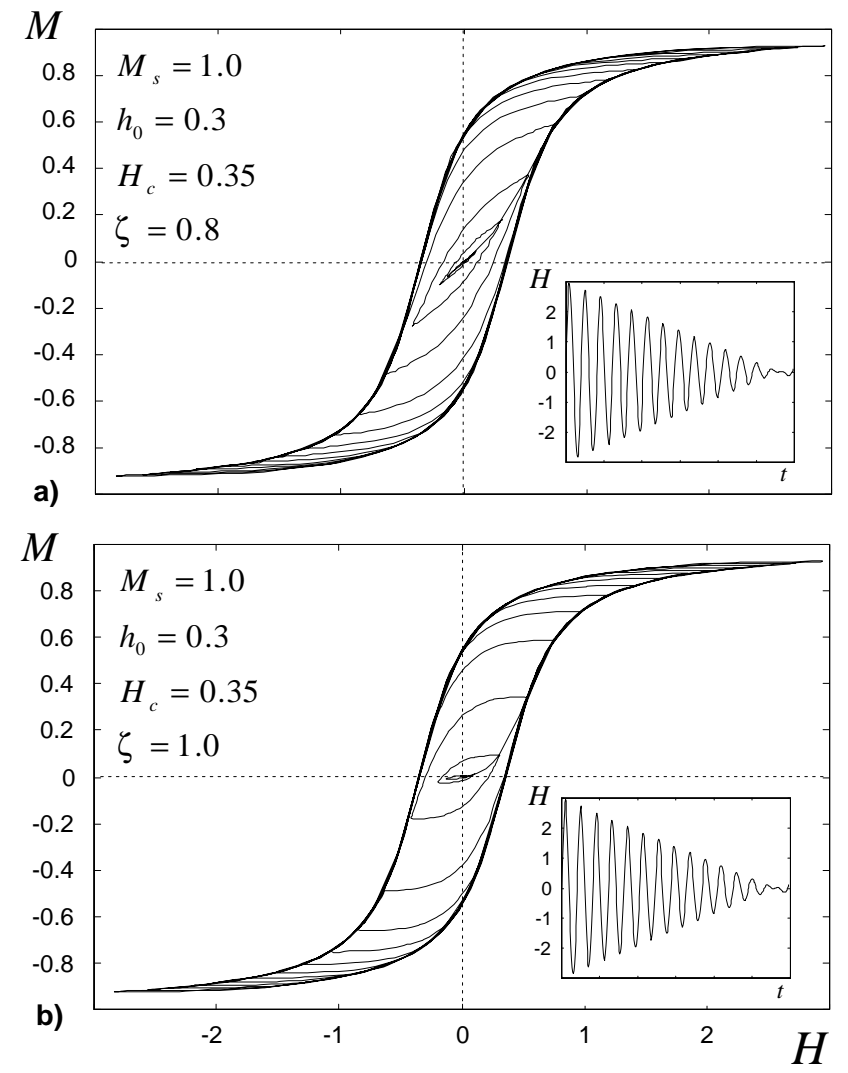

Figura 4: Simulação de histerese para dois conjuntos distintos de parâmetros utilizando o modelo proposto.

Preisach (Mayergoyz, 1991; Brokate e Sprekels, 1996). Duas regras básicas determinam a evolução da seqüência de memória com o tempo: uma regra que introduz novos pontos de reversão e outra que remove alguns dos pontos existentes. A primeira regra: no instante $t_{r}$, correspondente a uma reversão em $H, \delta$ sofre uma mudança abrupta, e um novo mínimo local $H_{i}^{\text {min }}=H\left(t_{r}\right)$ ou máximo local $H_{j}^{\max }=H\left(t_{r}\right)$ é armazenado na seqüência de memória. A segunda regra: se em qualquer instante $t$, $\delta=+1$ e $H(t)>H_{j}^{\max }$ ou $\delta=-1$ e $H(t)<H_{i}^{\min }$, o último par de valores extremos $\left(H_{i}^{\min }, H_{j}^{\max }\right)$, préexistentes na seqüência, é removido (wiped out). A seqüência discreta de memória pode ser representada por

$$
\mathbf{s}=\left(H_{0}^{\min }, H_{1}^{\min }, \ldots, H_{i}^{\min }, H_{j}^{\max }, \ldots, H_{1}^{\max }, H_{0}^{\max }\right) .
$$

Cada novo mínimo local $H_{i}^{\text {min }}$ ou máximo $H_{j}^{\max }$ de s é introduzido individualmente e removidos em pares $\left(H_{i}^{\min }, H_{j}^{\max }\right)$. As regras mencionadas anteriormente são atribuídas a Madelung (Brokate e Sprekels, 1996) e estão relacionadas à propriedade de retorno ao ponto de 


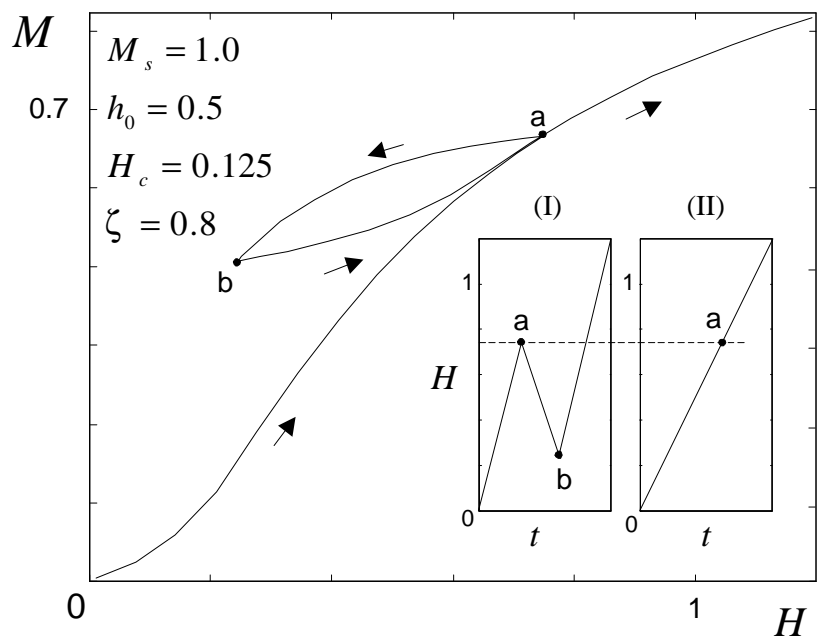

Figura 5: Teste por simulação das propriedades de memória relativas ao fechamento de laços menores e remoção de extremos para o modelo proposto.

partida do modelo de Preisach. A seqüência s permite ao modelo de Preisach "lembrar" recursivamente os valores do campo magnético nos instantes de reversão $H\left(t_{r}\right)$ (return-point memory), e também "esquecer" os mesmos quando apropriado (wiping-out property).

Vajda e Torre (1993) propôs uma representação na forma de equação diferencial para o modelo de Preisach

$$
\frac{d M_{i r r}}{d H}=\int_{H_{l}}^{H} \mu(\alpha, \beta) d \varepsilon
$$

sendo $d \varepsilon=d \alpha$ se $\delta=+1$ ou $d \varepsilon=d \beta$ se $\delta=-1$, e o limite inferior da integral $H_{l}$ é o valor do campo magnético correspondente à última reversão em $H(t)$ e é fornecido a (16) através da seqüência s. Assim, o modelo clássico de Preisach pode ser representado na forma diferencial como

$$
\frac{d M_{i r r}}{d H}=\left.F\left(H, H_{l}, \delta\right)\right|_{\mathbf{s} \rightarrow H_{l}},
$$

sendo $F\left(H, H_{l}, \delta\right)$ a função que representa a integral no lado direito de (16). Os valores de $H$, correspondentes aos pontos de reversão passados, aparecem em $H_{l}$ via s. Assim, o modelo Preisach inclui informação sobre a evolução passada da excitação $H$ através da seqüência s. Esta característica atribui ao modelo de Preisach a denominação de modelo de histerese com memória nãolocal.

A principal diferença entre os modelos de Preisach and Jiles é a estrutura discreta de memória $\mathbf{s}$ associada ao modelo de Preisach. Como conseqüência, o modelo de
Preisach assume a característica de persistência de memória não-local. Quando se assume que os domínios (representados pelos operadores elementares) não interagem, é possível haver persistência de memória. Na prática, os domínios interagem entre si e, dependendo do nível de interação e viscosidade (Bertotti, 1999), a seqüência $\mathbf{s}$ é parcialmente perdida e a propriedade de retorno ao ponto de partida não se mantém. Este fato resulta no fenômeno de acomodação (Torre, 1994). Na verdade, não há evidência experimental da persistência de memória não-local em materiais reais.

Para o modelo proposto, a equação (10) pode também ser representada na forma diferencial

$$
\frac{d M}{d H}=2 \frac{M_{s}}{\pi} \frac{h_{0}\left(1+P^{\prime}\left(\frac{H-H_{r}}{H_{p r}}\right)\right)}{h_{0}^{2}+\left(H_{p r} P\left(\frac{H-H_{r}}{H_{p r}}\right)+H-\delta H_{c}\right)^{2}},
$$

sendo

$$
P^{\prime}(x)=\left\{\begin{array}{cl}
-\zeta \cos \zeta x & , \zeta x<\frac{\pi}{2} \\
0 & , \zeta x>\frac{\pi}{2}
\end{array} .\right.
$$

Desde que $H_{p r}$ depende de $M_{r}$, a equação (18) pode ser representada na seguinte forma

$$
\frac{d M}{d H}=f\left(H, H_{r}, M_{r}, \delta\right)
$$

Assim, a seqüência de memória $\mathbf{s}$ associada ao modelo de Preisach, é reduzida no modelo proposto simplesmente ao valor $H_{r}$, correspondente ao último ponto de reversão. Os valores da excitação $H$ nos pontos de reversão $H_{r-1}, H_{r-2}, \ldots$ são incorporados no modelo através da inclusão da última magnetização de reversão $M_{r}$. A informação sobre a evolução passada de $H$ é incluída não apenas através do estado atual $\left(H_{0}, M_{0}\right)$, como no modelo de Jiles, mas também através de último estado de reversão $\left(H_{r}, M_{r}\right)$. Finalmente, a memória do modelo proposto é mais curta que a do modelo de Preisach e relativamente mais representativa que a do modelo de Jiles.

\section{DISCUSSÃO E RESULTADOS}

Pode ser verificado na equação (18) do modelo proposto, que a magnetização $M$ depende da definição de $F_{L}(\cdot)$ e $P(\cdot)$, e é determinada pelas coordenadas tanto do ponto atual $\left(H_{0}, M_{0}\right)$ quanto do último ponto de reversão $\left(H_{r}, M_{r}\right)$. A magnetização $M_{r}$, correspondente ao campo de reversão $H_{r}$, depende de estados prévios de reversão $\left(H_{r-1}, M_{r-1}\right)$, e assim por diante. Isto implica em um tipo de formação de memória que depende não apenas do estado atual $\left(H_{0}, M_{0}\right)$ mas também de $\left(H_{r}, M_{r}\right)$. Assim, é possível passar infinitas trajetórias 

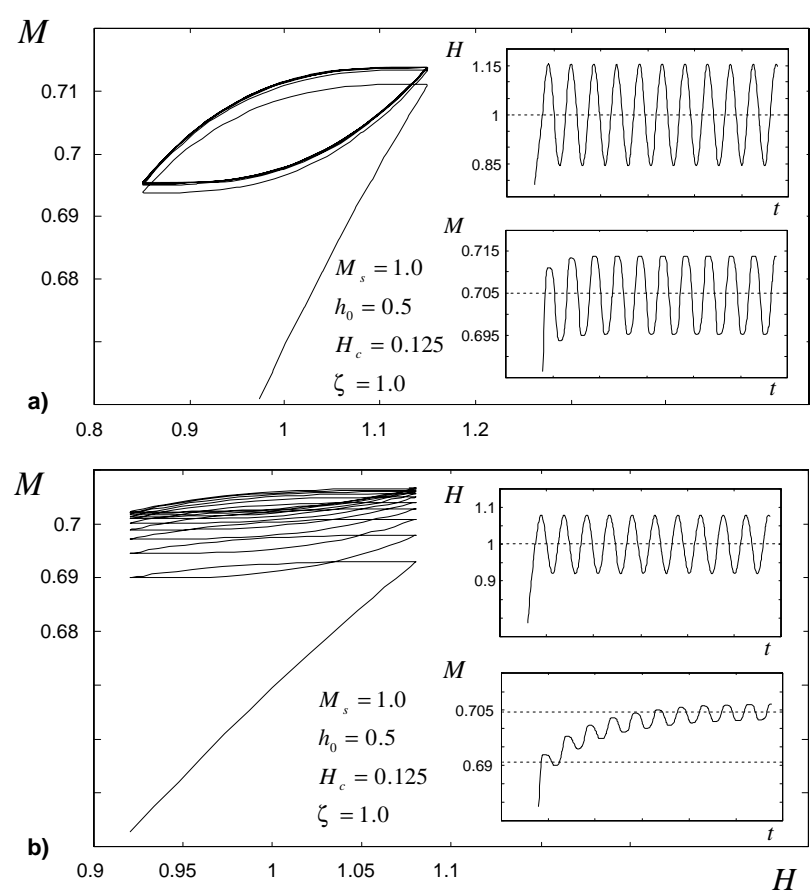

Figura 6: De forma similar a resultados experimentais usuais, os resultados de simulação com o modelo proposto demonstram que a acomodação depende da amplitude da excitação de campo: a) laço pequeno b) laço muito pequeno.

através do ponto $\left(H_{0}, M_{0}\right)$, cada uma delas correspondendo a um ponto particular $\left(H_{r}, M_{r}\right)$ dentro do laço principal.

Em contraste com a equação diferencial (18), o modelo algébrico (10) demanda uma carga computacional que depende apenas do número de pontos escolhidos para representar a curva de histerese, e isto não afeta a precisão dos cálculos para determinar o valor de $M$.

O desempenho do modelo é avaliado em termos da capacidade do mesmo em exibir propriedades das nãolinearidades genéricas da histerese magnética. Uma vez que estas propriedades sejam satisfeitas, espera-se que a identificação correta dos parâmetros permita que o modelo proposto represente não-linearidades observadas experimentalmente.

Nas figuras 4 (a) e (b) o efeito do parâmetro $\zeta$ na forma da curva de histerese e na capacidade do modelo de gerar laços menores aninhados, são apresentados para uma excitação dada por

$$
H(t)=3 \sin (0.85 t)(-0.01 t+1) .
$$

A susceptibilidade incremental para $\zeta=0.8$ é maior do

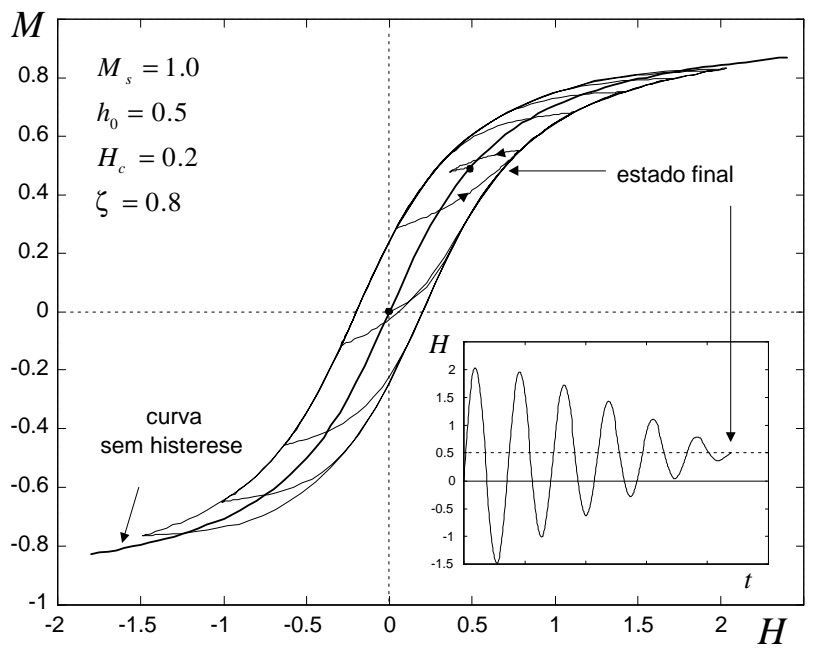

Figura 7: Comportamento do modelo proposto, excitado com um campo de desmagnetização alternado e um campo contínuo superposto $H_{d c}=0.5$. O estado final de desmagnetização recai sobre um ponto da curva sem histerese $M_{L}\left(H_{d c}, \delta=0\right)$.

que para $\zeta=1.0$.

A propriedade de retorno ao ponto de partida e a propriedade de remoção estão fortemente relacionadas, e para o modelo proposto as mesmas só podem ser avaliadas qualitativamente e aproximadamente por simulação, desde que estas propriedades são na verdade características do modelo de Preisach. Em curvas de histerese observadas experimentalmente, existe o fenômeno de acomodação e estas duas propriedades só são satisfeitas aproximadamente. Na figura 5 é apresentada a resposta do modelo proposto quando submetido a duas formas de onda diferentes do campo $H$ (Figura 5, quadros interiores I e II). No quadro interior I é mostrada a forma de onda do campo $H$ utilizada para gerar um laço menor. A trajetória inicia em $(H=0, M=0)$, cresce monotonicamente até $a$, decresce até $b$ e cresce novamente. Para a forma de onda de excitação $H$ apresentada no quadro interior II, a trajetória traçada no plano $M-H$ é aproximadamente a mesma da traçada como resultado da excitação com a forma de onda do quadro interior I, com exceção do laço menor. Nota-se que o laço menor inicia e termina aproximadamente no mesmo ponto (return-point memory property).

Para laços menores com largura muito menor do que a largura do laço principal, o fenômeno de acomodação se torna evidente (Figura 6). Quando a amplitude de excitação é 0.3 unidades (Figura 6a), o laço menor se torna estável após o segundo ciclo. Se a amplitude da 


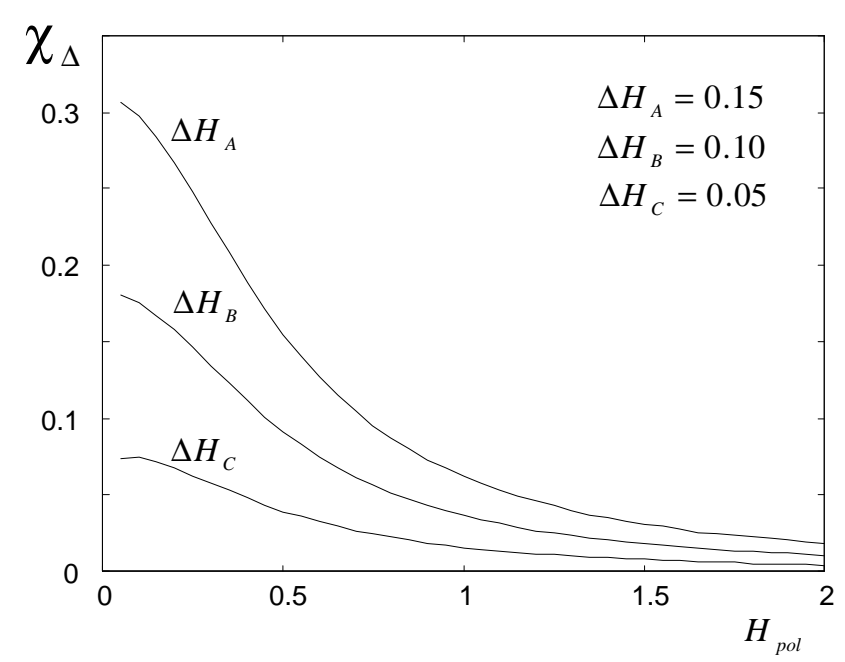

Figura 8: Susceptibilidade incremental calculada utilizando o modelo proposto. Obtida para valores diferentes de largura do laço menor $\Delta H$, em função do campo de polarização $H_{p o l}$.

excitação é 0.15 unidades, o processo de acomodação leva vários ciclos (Figura 6 b). Este fenômeno pode ser também observado através da resposta $M(t)$ (quadros interiores das Figuras 6a e b).

Na Figura 7 observa-se que se a excitação é um campo oscilatório desmagnetizante com um valor médio $H_{\text {pol }}$ de 0.5 unidades (quadro interior), o estado final resultante é um ponto na curva sem histerese $F_{L}(H, \delta=0)$. Seguindo este mesmo procedimento para valores diferentes de $H_{\text {pol }}$, é possivel traçar completamente a curva sem histerese $F_{L}(H, \delta=0)$.

Finalmente, é apresentada na Figura 8 a susceptibilidade incremental calculada utilizando o modelo proposto, e obtida como resultado de excitações de pequena amplitude para valores diferentes de $H_{\text {pol }}$. A susceptibilidade incremental é determinada somente quando o laço menor se torna estável, após o transitório inicial. $\mathrm{O}$ valor de $\chi_{\Delta}$ decresce com o aumento de $H_{\text {pol }}$. Além disso, o valor de $\chi_{\Delta}$ também depende da amplitude $\Delta H$. Estas características são muito similares às observadas em transdutores magnéticos reais.

\subsection{Aplicação do Modelo Proposto para Ferrite tipo MnZn}

A aplicação do modelo proposto é ilustrada utilizando a relação entre densidade de fluxo $B$ e campo magnético $H$. Para materiais tipo MnZn, a densidade de fluxo e a magnetização são equivalentes. Baseando-se na equação

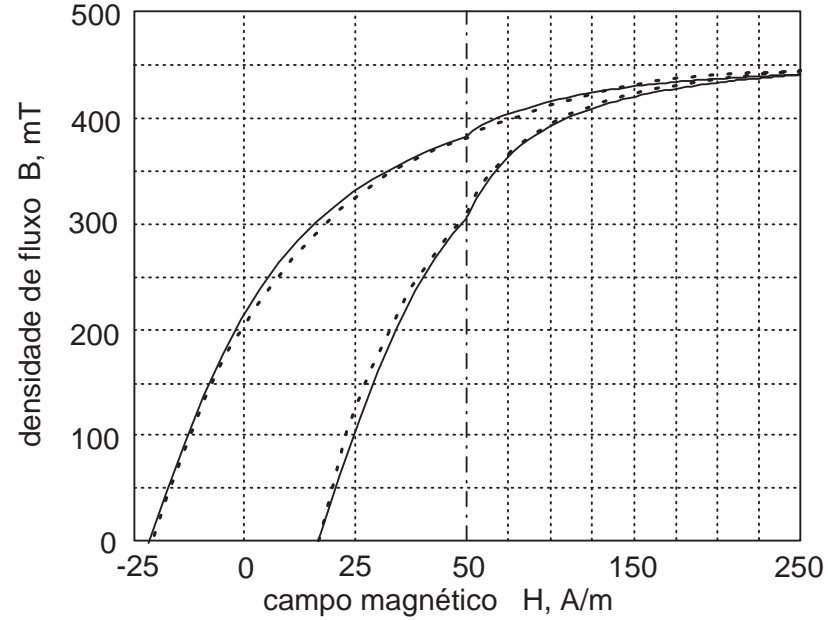

Figura 9: Simulação com o modelo proposto (linha sólida) ajustado para dados experimentais (www.ferroxcube.com) do ferrite MnZn 3c15 (linha tracejada).

(10), escreve-se a relação $B \times H$ na forma

$$
B(H)=\frac{2 B_{s}}{\pi} \arctan \left(\frac{H_{p r} P\left(\frac{H-H_{r}}{H_{p r}}\right)+H-\delta H_{c}}{h_{0}}\right),
$$

sendo $B_{s}$ a densidade de fluxo de saturação. Para ilustrar a generalidade da proposta, foi escolhida uma função de proximidade diferente da apresentada na equação (11), dada por

$$
P(x) \triangleq \tanh (-\zeta x)+1
$$

sendo que o parametro $\zeta$ define como as trajetórias internas se aproximam do laço principal.

Para validar experimentalmente o modelo proposto, foi selecionado o ferrite MnZn 3c15 fabricado pela Ferroxcube. Os parâmetros obtidos para este material são $H_{c}=18 \mathrm{~A} / \mathrm{m}, B_{s}=0.47 \mathrm{~T}, h_{0}=23$ and $\zeta=0.8$. $\mathrm{Na}$ Figura 9, o primeiro quadrante do laço principal da característica $B \times H$ do ferrite $3 C 15$ é comparado com o correspondente obtido com o modelo proposto. Observa-se uma concordância razoável entre dado experimental e simulação.

Para enfatizar a simplicidade da implementação numérica, é apresentado a seguir um script MATLAB para calcular a desmagnetização do ferrite 3c15, obtido através de um campo senoidal decrescente. O resultado deste processo de desmagnetização, com um fluxo residual $B_{H=0}=0.14 \mathrm{~T}$, é apresentado na Figura 10. O intervalo $d$ pode ser arbitrariamente escolhido sem o cuidado necessário quando se escolhe o passo de integração 


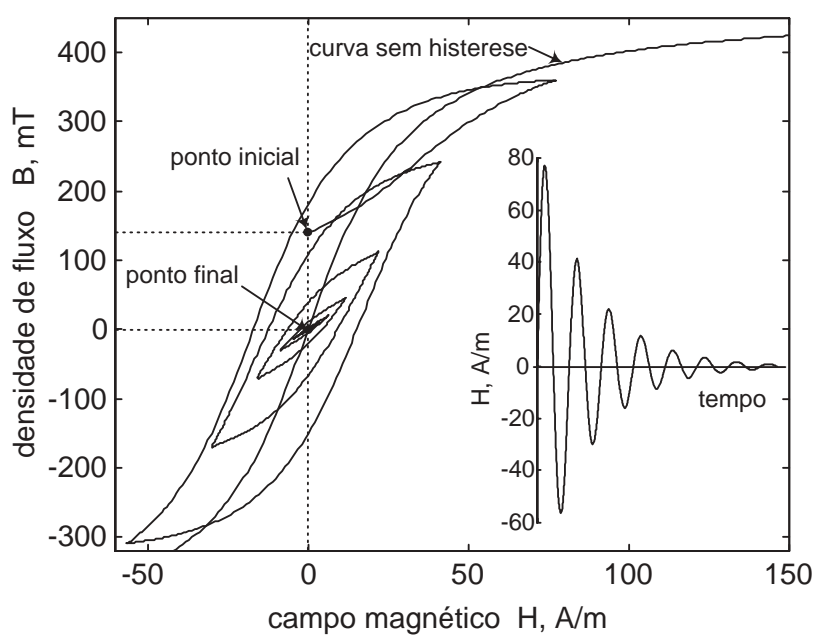

Figura 10: Processo de desmagnetização do ferrite MnZn 3c15 simulado com o modelo proposto.

na solução de equações diferenciais. Para o modelo algébrico proposto, a precisão numérica da histerese resultante é independente do intervalo $d$, e o custo computacional pode ser consideravelmente reduzido quando comparado a solução de equações diferenciais.

\subsubsection{Código MATLAB}

$\mathrm{Hc}=18 ; \mathrm{Bs}=0.47 ; \mathrm{h} 0=23 ;$ zeta=0.8; d=0.02;

$t=0: d: 15 * p i ; H=90 * \sin (t) . * \exp (-t / 10)$;

$\operatorname{delta}=2 *([0 \operatorname{diff}(H)]>0)-1$;

reversal $=[\operatorname{diff}(\operatorname{delta}) 0]$;

$\mathrm{Hr}=\mathrm{H}(1) ; \mathrm{Br}=0.14 ; \mathrm{B}=0.14$;

for $n=1: 1: \operatorname{length}(H)$,

$\mathrm{Hpr}=\mathrm{h} 0 * \tan (\mathrm{pi} * \mathrm{Br} /(2 * \mathrm{Bs}))+\operatorname{delta}(\mathrm{n}) * \mathrm{Hc}-\mathrm{Hr}$;

$\mathrm{Hp}=\mathrm{Hpr} *(\tanh (-\operatorname{zeta} *(\mathrm{H}(\mathrm{n})-\mathrm{Hr}) / \mathrm{Hpr})+1)$;

$\mathrm{B}(\mathrm{n})=2 *(\mathrm{Bs} / \mathrm{pi}) * \operatorname{atan}((\mathrm{H}(\mathrm{n})-$

$\operatorname{delta}(\mathrm{n}) * \mathrm{Hc}+\mathrm{Hp}) / \mathrm{hO})$;

if $\operatorname{reversal}(n)^{\sim}=0$,

$\mathrm{Hr}=\mathrm{H}(\mathrm{n}) ; \mathrm{Br}=\mathrm{B}(\mathrm{n})$;

end

end

$\operatorname{comet}(\mathrm{H}, 1000 * \mathrm{~B})$

\section{CONCLUSÃO}

Um novo modelo para representação da histerese magnética foi apresentado neste trabalho. Definiu-se o conceito de proximidade ao laço principal, a partir do qual uma equação algébrica foi derivada para representar matematicamente a histerese em materiais magnéticos. Esta representação tem a vantagem de não necessitar da solução de equação diferencial ou de integral dupla. O custo computacional é então consideravelmente reduzido quando comparado ao modelo de Jiles e especialmente ao modelo de Preisach.

O modelo proposto é independente da taxa e apenas quatro parâmetros são necessários para obtenção de $M(H)$. Um procedimento relativamente simples de estimação de parâmetros foi apresentado. As propriedades do modelo foram investigadas em termos de nãolinearidades bem conhecidas da histerese magnética. O modelo proposto foi capaz de descrever tais nãolinearidades, incluindo: laços maior e menores, acomodação, curva inicial de magnetização e curva sem histerese.

\section{AGRADECIMENTOS}

Os autores agradecem o apoio da CAPES, do CNPq/PRONEX e do DAAD (German Academic Exchange Service), na forma de bolsas de estudo e pesquisa, durante a realização dessa investigação.

\section{REFERÊNCIAS}

Basso, V. e Bertotti, G. (1996). Hysteresis models for the description of domain wall motion, IEEE Transactions on Magnetics 32(5): 4210-4212.

Basso, V., Bertotti, G., Serpico, C. e Visone, C. (1998). Application of an exactly invertible hysteresis model to magnetic field computations, Journal de Physique IV 8(2): 639-642.

Bertotti, G. (1999). Hysteresis in Magnetism, Academic Press.

Brokate, M. e Sprekels, J. (1996). Hysteresis and Phase Transitions, Springer-Verlag.

de Almeida, L., Deep, G., Lima, A., Neff, H. e Freire, R. (2000). A hysteresis model for vanadium oxide thermal radiation sensor, Proceedings of the 17th IEEE Instrumentation and Measurement Technology Conference - IMTC. 
Hodgdon, M. L. (1988). Mathematical theory and calculations of magnetic hysteresis curves, IEEE Transactions on Magnetics 24(6): 3120-3122.

Jiles, D. e Atherton, D. (1983). Ferromagnetic hysteresis, IEEE Transactions on Magnetics 19(5): 21832185 .

Krasnoselskii, M. A., Pokrovskii, A. e Niezgodka, M. (1989). Systems With Hysteresis, Springer-Verlag.

Mayergoyz, I. (1991). Mathematical Models of Hysteresis, Springer-Verlag.

Ohta, T., Enomoto, Y. e Kato, R. (1991). Domain growth with time-dependent front velocity in one dimension, Physiscal Review B 43(16): 262-268.

Preisach, F. (1935). Uber die magnetische nachwrikung, Zeitschrift fur Physik 94: 277-302.

Saliah, H. e Lowther, D. (1997). The use of neural network in magnetic hysteresis identification, Physica B 233(4): 318-323.

Serpico, C. e Visone, C. (1998). Magnetic hysteresis modeling via feed-forward neural networks, IEEE Transactions on Magnetics 34(3): 623-628.

Torre, E. D. (1991). Existence of magnetizationdependent preisach models, IEEE Transactions on Magnetics 27(4): 3697-3699.

Torre, E. D. (1994). A preisach model for accommodation, IEEE Transactions on Magnetics 30(5): 2701-2707.

Torre, E. D. (1999). Magnetic Hysteresis, IEEE Press.

Vajda, F. e Torre, E. D. (1993). Efficient numerical implementation of complete-moving-hysteresis models, IEEE Transactions on Magnetics 29(2): 15321537 .

Visintin, A. (1994). Differential Models of Hysteresis, Springer-Verlag. 